\title{
Compact hyperspectral instruments
}

Paul Jougla, Alice Robert, Jérémie Lochard, Joel Boyadjian, Vincent Costes, et al.

Paul Jougla, Alice Robert, Jérémie Lochard, Joel Boyadjian, Vincent Costes, Laurie Pistre, Matthieu Castelnau, Damien Rodat, Christian Imbert, Camille Desjardins, "Compact hyperspectral instruments," Proc. SPIE 11852, International Conference on Space Optics - ICSO 2020, 118523J (11 June 2021); doi: 10.1117/12.2599609

SPIE Event: International Conference on Space Optics - ICSO 2021, 2021, Online Only 


\section{International Conference on Space Optics-ICSO 2020}

Virtual Conference

30 March-2 April 2021

Edited by Bruno Cugny, Zoran Sodnik, and Nikos Karafolas
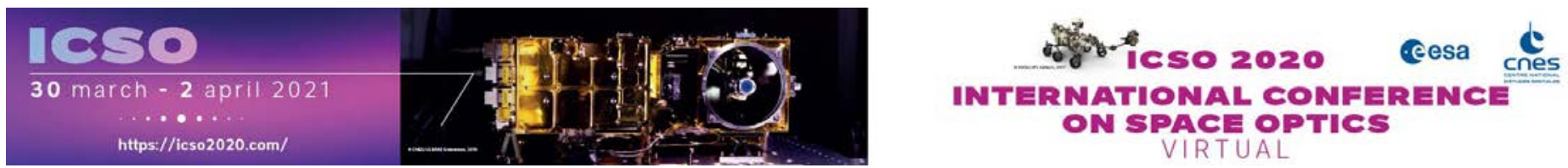

\section{Compact hyperspectral instruments}

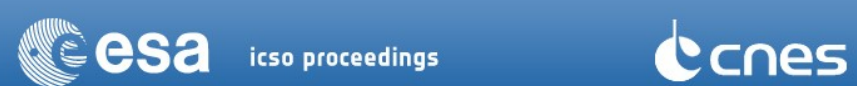

International Conference on Space Optics - ICSO 2020, edited by Bruno Cugny, Zoran Sodnik, Nikos Karafolas, Proc. of SPIE Vol. 11852, 118523J · (c) 2021 ESA and CNES

CCC code: $0277-786 \mathrm{X} / 21 / \$ 21 \cdot$ doi: $10.1117 / 12.2599609$ 


\title{
COMPACT HYPERSPECTRAL INSTRUMENTS
}

\author{
Paul Jougla $^{* a}$ (paul.jougla@airbus.com), Alice Robert ${ }^{\mathrm{a}}$ (alice.robert@airbus.com), Jérémie Lochard ${ }^{\mathrm{a}}$ \\ (jeremie.lochard@airbus.com), Joël Boyadjian (joel.boyadjian@airbus.com) \\ Vincent Costes ${ }^{\mathrm{b}}$, Laurie Pistre ${ }^{\mathrm{b}}$, Matthieu Castelnau ${ }^{\mathrm{b}}$, Damien Rodat ${ }^{\mathrm{b}}$, Christian Imbert $^{\mathrm{b}}$ \\ Camille Desjardins \\ aAirbus D\&S France - 31, Av. des Cosmonautes - 31402 Toulouse Cedex 4- France \\ ${ }^{\mathrm{b}} \mathrm{CNES}-18$, avenue Edouard Belin - 31401 Toulouse Cedex 9
}

\begin{abstract}
This presented study is initiated in the frame ofCNES advanced studies. It aims at providing a better understanding of driving parameters for this type of instruments, and exploring suitable, very compact hyperspectral ins truments based on grating spectrometers.

First a scalar model for multiblazed gratings is developed, and confronted with more detailed diffraction models.

The spectral band is in the range of $400-2500 \mathrm{~nm}$, with a spectral resolution of about $10 \mathrm{~nm}$ width. The Ground Sampling Distance (GSD) shall be between 10 and $15 \mathrm{~m}$, the Field of View (FOV) shall be larger than $8 \mathrm{~km}$, the orbit Height is $550 \mathrm{~km}$.

Three types of instruments are proposed, a common spectrometer with a unique detector, a common spectrometer working in diffraction order 1 for the SWIR, and 2 for the VIS spectral band, and 2 spectrometers within field separa tion.

For each instrument the grating blazed profile is optimized, the key instruments parameters computed, and an optical configuration is proposed.

The study shows compactness optimization with these three instrument concepts, thanks to an entrance pupil diameter reduction along with better grating diffraction efficiency
\end{abstract}

Keywords: hyperspectral, spectrometer, grating, multiblazed, scalar diffraction model, freeform

\section{INTRODUCTION}

Hypers pectral instruments for Earth observation are designed for providing many multispectral images, typically in the range of 200 spectral bands.

This presented study is initiated in the frame ofCNES advanced studies. It aims at providing a better understanding of driving parameters for this type of instruments, and exploring suitable, very compact and relevant solutions for the following mission:

The spectral band is in the range of $400-2500 \mathrm{~nm}$, with a spectral resolution of about $10 \mathrm{~nm}$ width. The Ground Sampling Distance (GSD) shall be between 10 and $15 \mathrm{~m}$, the Field of View (FOV) shall be larger than $8 \mathrm{~km}$, the orbit Height is $550 \mathrm{~km}$. A good SNR is requested for the VIS and SWIR wavelengths, considering the following reference luminances:

\begin{tabular}{|l|r|r|r|r|}
\hline wavelength $(\mathrm{nm})$ & 600 & 860 & 1600 & 2200 \\
\hline Lref $(\mathrm{W} / \mathrm{m} 2 / \mu \mathrm{m} / \mathrm{sr})$ & 70 & 70 & 15 & 5 \\
\hline
\end{tabular}

The goal is to design the most compact hyperspectral imager, preferably based on a grating s pectrometer.

First a pre-sizing has been performed according to the available detectors, and then grating diffraction efficiency is studied with a scalar model. It demonstrates the need of a multiblazed grating profile to optimize grating diffraction efficiency at the longest wavelengths. 
The instrument is made of a telescope, with one or two slits on its focal plane. It is followed by one or two grating spectrometer, either Dyson or Offner (cf. [1], [2], [3]). The different images of the slit in the various wavelengths are imaged on the Focal Plane Array (FPA).

The following three options are studied for the spectrometer:

- $\quad$ A unique spectrometer covering the whole waveband with the diffraction order number 1

- $\quad$ A unique spectrometer, which uses diffraction order 1 for the SWIR wavebands, and diffraction order 2 for the visible wavelengths

- $\quad$ Two separate spectrometers, for the VIS and for the SWIR sub wavebands.

\section{Choice of the detector:}

MCT is needed if a single detector is implemented to cover the whole spectral range. In case of spectral splitting and use of two detectors to cover the whole spectral range, the use of a silicon detector for VNIR band and a MCT one for SWIR band may be preferred for cost reason. Nevertheless, a pixel pitch of $18 \mu \mathrm{m}$ is considered for this study, which can be the result of a macropixel if silicon pixel pitch is found too small.

\section{GRATING PROFILE OPTIMIZATION}

The gratings of all the instruments encountered in this study have a period much larger than wavelengths. Grating diffraction efficiency can therefore be best computed with a simplified scalar model.

The Optical Path Difference (OPD) added by the blazed grating to the electrical field phase is given in figure below:

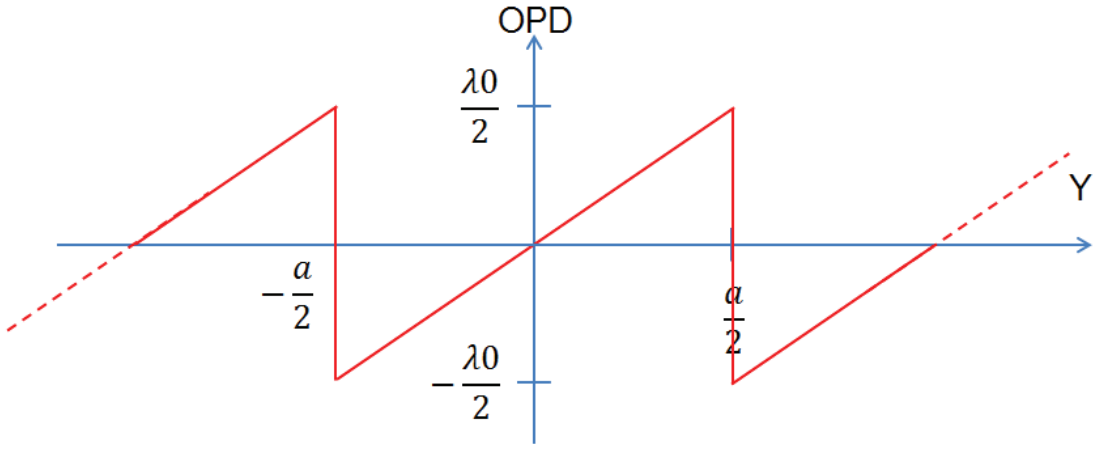

Figure 1. Optical path difference (OPD) added by a mono blazed grating

An incident wave plane is diffracted in several diffraction orders.

Intensity in the kth diffraction order is given by the classical formula:

$$
I_{k}^{\prime}=\operatorname{sinc}^{2}\left(\pi \cdot\left(\frac{\lambda 0}{\lambda}-k\right)\right)
$$

In order to balance efficiency in VNIR and SWIR parts of the spectrum the best compromise is to choose the blazed wavelength equal to $700 \mathrm{~nm}$ : 


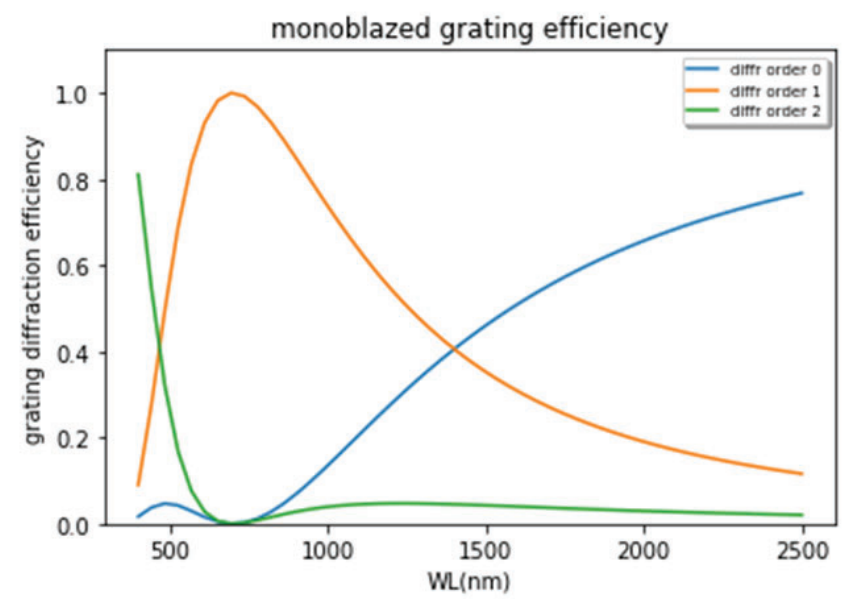

Figure 2. Diffraction efficiency of a monoblazed grating at $700 \mathrm{~nm}$, scalar model

Wavelength range covers more than 2 decades: a monoblazed grating is not capable of a high efficiency over such a large range of wavelengths, transmission of the instrument would be lower than $10 \%$ at the 2 edges of the spectrum, leading to very low SNR. This is critical for the SWIR wavelengths, for which the luminance tends to be lower than in the visible part of the spectrum.

In order to optimize the transmission all over the instrument spectrum it is necessary to design a multiblazed grating profile (ref [4]).

We develop here a dedicated scalar model of such a multiblazed grating, in order to optimize the grating profile and the instrument transmission.

The optical path difference added by the grating is a function with periodicity a, made of $\mathrm{N}$ segments with number $\mathrm{j}=1$ to $\mathrm{N}$

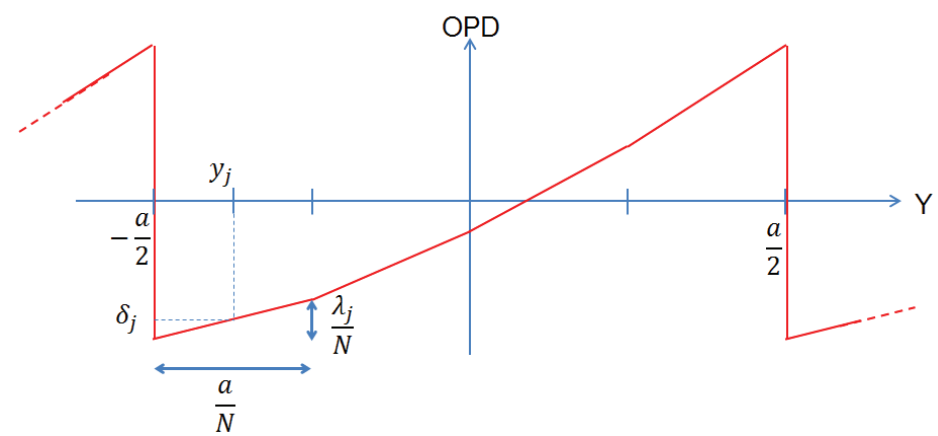

Figure 3. Multiblazed grating profile: the profile is made of $\mathrm{N}$ segments

On segment number $\mathrm{j}$ the OPD is given by:

$$
O P D_{j}(y)=\frac{y-y_{j}}{a} \cdot \lambda_{j}+\delta_{j}
$$

( $\lambda_{j}$ would be the blazed wavelength in the case this segment was extended to the whole period a of the grating.) 
We arbitrarily choose $\delta_{1}=0$, and we constrain the profile to be a continuous function, for manufacturability reasons. Therefore $\delta_{j+1}=\delta_{j}+\frac{\lambda_{j}+\lambda_{j+1}}{2}$.

The multiblazed grating profile is therefore completely described by the set of all the $\lambda_{j}$, for $\mathrm{j}=1$ to $\mathrm{N}$.

Let's consider a plane wave incident on the grating at normal incidence. The grating is on the (x,y) plane and the incident wave propagates along the $\mathrm{Z}$ axis. The grating grooves are aligned with $\mathrm{X}$ axis.

The incident electrical field is described by: $E(x, y)=1$. After the grating the electrical field is $E(x, y)=e^{i \cdot O P D(y)}$ As $\operatorname{OPD}(y)$ is a-periodic, the Fourier series coefficients give the electrical field at infinite.

We therefore compute the Fourier transform for each of the $\mathrm{N}$ segments, and then add them coherently to get the electrical field in diffraction order k:

$$
E_{k}^{\prime}=\frac{1}{N} \sum_{j=1}^{N} e^{i 2 \pi\left(\frac{\delta_{j}}{\lambda}-k \frac{y_{j}}{a}\right)} \cdot \operatorname{sinc}\left(\frac{\pi}{N} \cdot\left(\frac{\lambda_{j}}{\lambda}-k\right)\right)
$$

Intensity is then given by the square of the norm of the electrical field:

$$
I_{k}^{\prime}=E_{k}^{\prime} \cdot \overline{E_{k}^{\prime}}
$$

We used Python to optimize the multiblazed grating profile for each of the applications described here after.

The goal is to design the ideal multi-blazed profile, which gives the best theoretically grating efficiency.

\section{INSTRUMENT OPTIMIZATION}

\subsection{Instrument with one grating spectrometer, used in the $1 \mathrm{st}$ diffraction order}

Grating optimization:

As mentioned before a monoblazed grating cannot cover efficiently the whole spectral band $[400,2500 \mathrm{~nm}]$ with diffraction order \#1: diffraction efficiency is $100 \%$ for the blazed wavelength, but drops on the 2 edges of the spectral band. The goal is to modify the grating blazed profile in order to balance efficiency all over the spectral band, at the expense of the peak value. More specifically we put more weight on the higher wavelengths, for which scene luminance is lower than for the VIS wavelengths.

This python optimization led to the profile shown below, and the related diffraction efficiency for the first diffraction orders: 

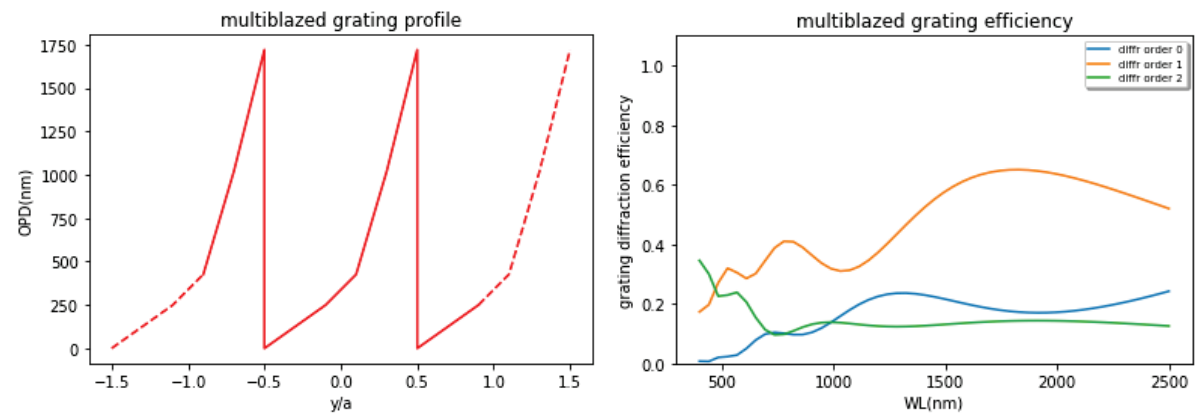

Figure 4. Multiblazed grating profile optimized for the $[400,2500 \mathrm{~nm}]$ spectral band, diffraction efficiency for this multiblazed grating. The five wavelengths used for the multiblazed profile are: $[623,623,883,2965,3504] \mathrm{nm}$

The simplified scalar model is very convenient to quickly optimize the grating profile. A more detailed model, such as the one used by software PC grate, is more realistic, and takes the polarization into account.

Figure below shows the two models fit correctly, and that the scalar approximation is valid as wavelength is smaller than grating period. At shorter wavelengths the two models do not agree well and further investigation shall be made in order to optimize the diffraction efficiency in this region. Ref [4] shows such a grating is possible.

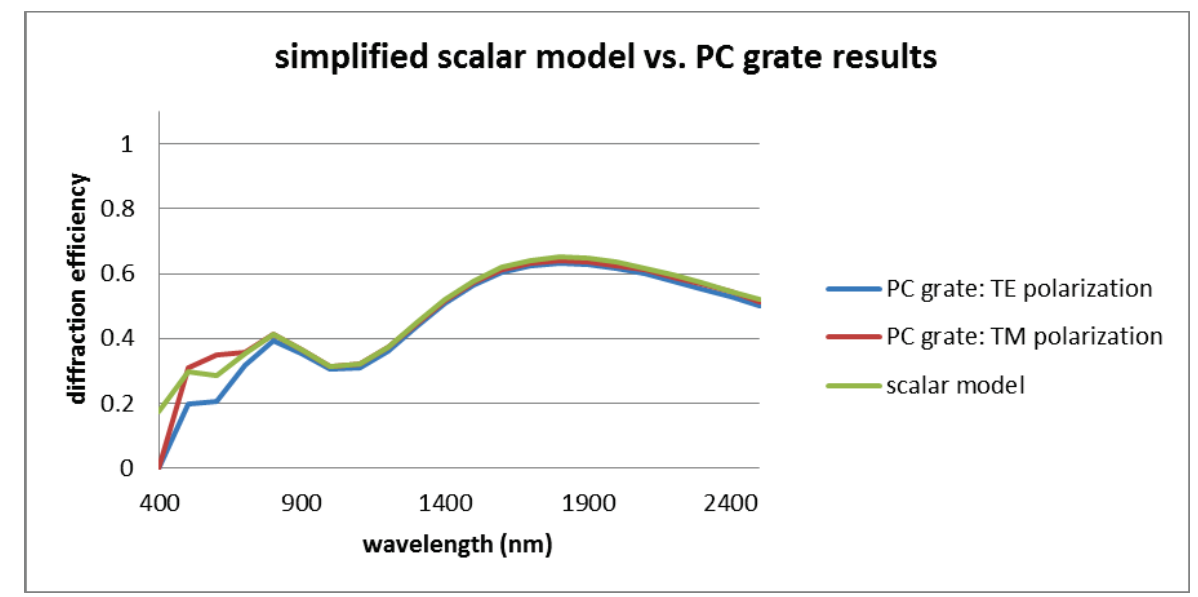

Figure 5. Multiblazed grating profile optimized for the $[400,2500 \mathrm{~nm}]$ spectral band: comparison of scalar model and PC grate model

Table 1. The instrument key parameters are calculated from SNR budget and taking into account the grating efficiency:

\begin{tabular}{|l|r|r|r|r|}
\hline wavelength $(\mathrm{nm})$ & 600 & 860 & 1600 & 2200 \\
\hline grating efficiency & 0.22 & 0.2 & 0.5 & 0.6 \\
\hline instrument transmission & 0.13 & 0.118 & 0.296 & 0.355 \\
\hline Lref $(\mathrm{W} / \mathrm{m} 2 / \mu \mathrm{m} / \mathrm{sr})$ & 70 & 70 & 15 & 5 \\
\hline EPD $(\mathrm{mm})\left(^{*}\right)$ & \multicolumn{5}{|c|}{350} \\
\hline SNR & OK & OK & OK & OK \\
\hline
\end{tabular}

$\left({ }^{*}\right)$ EPD $=$ Entrance Pupil Diameter 


\section{Optical design:}

In order to achieve the UV SNR specification a large pupil (diameter 350mm) has to be accomodated.

This leads to a very large telescope, whereas the spectrometer volume is much smaller, whatever the type of design: Dyson spectrometer or Offner spectrometer.

In order to optimize the telescope volume the best choice is to design a 3 mirrors telescope, with freeform mirrors.

In the case of the Offner spectrometer, the volume of the spectrometer is optimized with 2 freeform mirrors. The convex grating is ruled on a spherical surface: choosing a freeform grating marginally reduces the global volume of the instrument, and keeping a spherical grating is best for manufacturability.

As for the Dyson spectrometer a spherical grating permits a quite compact spectrometer. Aspherizing it does not help in reducing notably the spectrometer volume, which is , again, negligible vs. telescope volume.

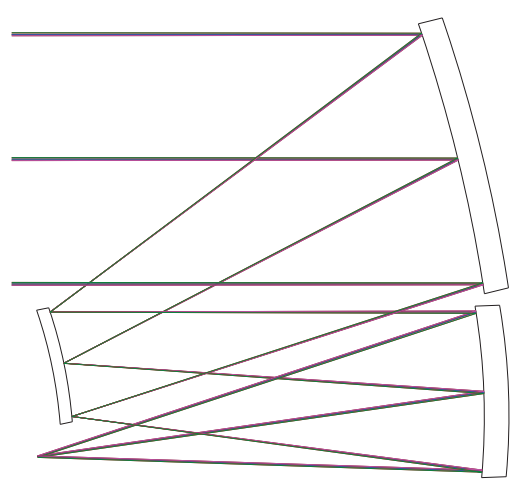

$100.00 \quad$ MM

Figure 6. Telescope for the first instrument option: 3 freeform mirrors, volume $=100$ liters

Table 2. Offner spectrometer and Dyson spectrometer comparison for the first instrument option

\begin{tabular}{|l|l|}
\hline Offner spectrometer & Dyson spectrometer \\
\hline $\begin{array}{l}\text { Mirror 2, } \\
\text { Mreeform }\end{array}$ \\
\hline freeform
\end{tabular}


An OSF (Order Sorting Filters) will be placed just before the FPA, in order to decrease the level of straylight due to neighbor grating diffraction orders.

The Dyson shall be folded before the FPA, in order to accommodate the detector.

The figure below gives a view of the telescope and the Offner spectrometer:

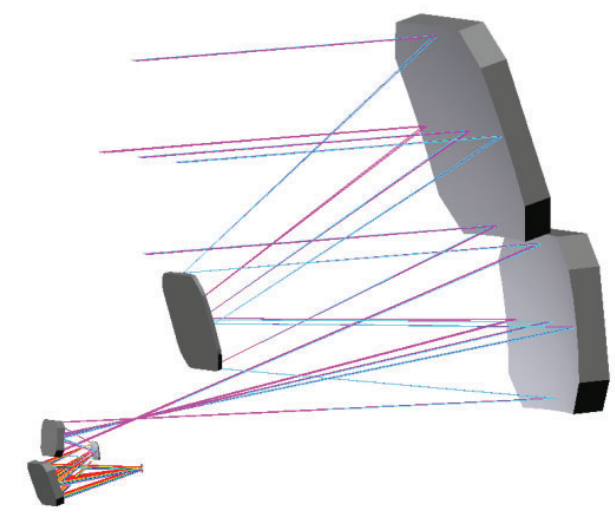

Figure 7. instrument for the first instrument option

\subsection{Instrument with one grating spectrometer, using two diffraction orders}

\section{Grating optimization}

The previous design volume is quite big: its large entrance pupil compensates the low grating diffraction efficiency.

As waveband covers more than 2 decades it is possible to dedicate shorter wavelengths to grating diffraction order 2, and longer ones to diffraction order 1 . The waveband is therefore split into two sub wavebands, imaged on two dedicated detectors. The limit between the 2 wavebands is fixed at $1000 \mathrm{~nm}$, so that a silicon detector is able to image lower wavelengths. With this option we get better grating diffraction efficiency:
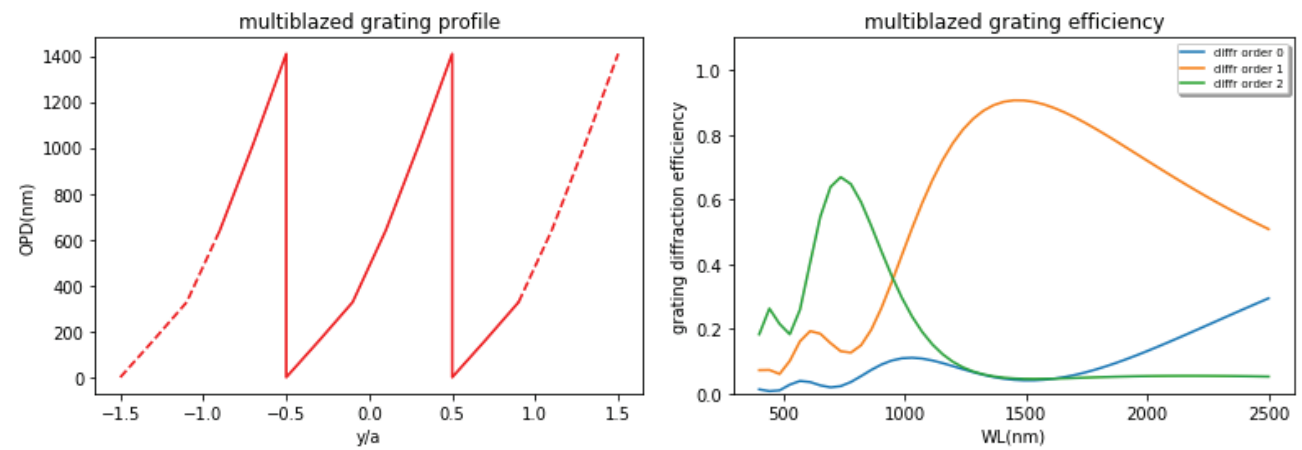

Figure 8. Multiblazed grating profile optimized for the $[400,2500 \mathrm{~nm}]$ spectral band. $[400,1000 \mathrm{~nm}]$ spectral band uses diffraction order number 2, [1000,2500nm] spectral band uses diffraction order number 1. On the right: Diffraction efficiency for this multiblazed grating. The five wavelengths used for the multiblazed profile are: $[807,829,1565,1869,1974] \mathrm{nm}$ 
As diffraction efficiency gets better in the SWIR waveband, it is possible to work with a smaller entrance pupil instrument: entrance pupil diameter is now $300 \mathrm{~mm}$ :

Table 3. The instrument key parameters are calculated from SNR budget and taking into account the grating efficiency:

\begin{tabular}{|l|r|r|r|r|}
\hline wavelength $(\mathrm{nm})$ & 600 & 860 & 1600 & 2200 \\
\hline grating efficiency & 0.35 & 0.45 & 0.5 & 0.65 \\
\hline instrument transmission & 0.187 & 0.24 & 0.266 & 0.346 \\
\hline Lref $(\mathrm{W} / \mathrm{m} 2 / \mu \mathrm{m} / \mathrm{sr})$ & 70 & 70 & 15 & 5 \\
\hline EPD $(\mathrm{mm})\left(^{*}\right)$ & \multicolumn{5}{|c|}{300} \\
\hline SNR & OK & OK & OK & OK \\
\hline
\end{tabular}

In this case, pupil size is well balanced between UV and SWIR SNR needs.

\section{Optical design:}

Telescope:

- Similar to the first solution: 3 freeform mirrors

- $\quad$ scale down: volume $=81$ liters

\section{Spectrometer:}

The two useful grating orders are superimposed over spectral range and split before focal planes using a Dichroic Beam Splitter(DBS). The DBS reflects wavelengths shorter than $1000 \mathrm{~nm}$, and transmits wavelengths larger than $1000 \mathrm{~nm}$. The two sub wavebands are then imaged on a dedicated detector.

The spectrometer is an Offner spectrometer. Indeed it would be very difficult to accommodate the DBS in a Dyson spectrometer:

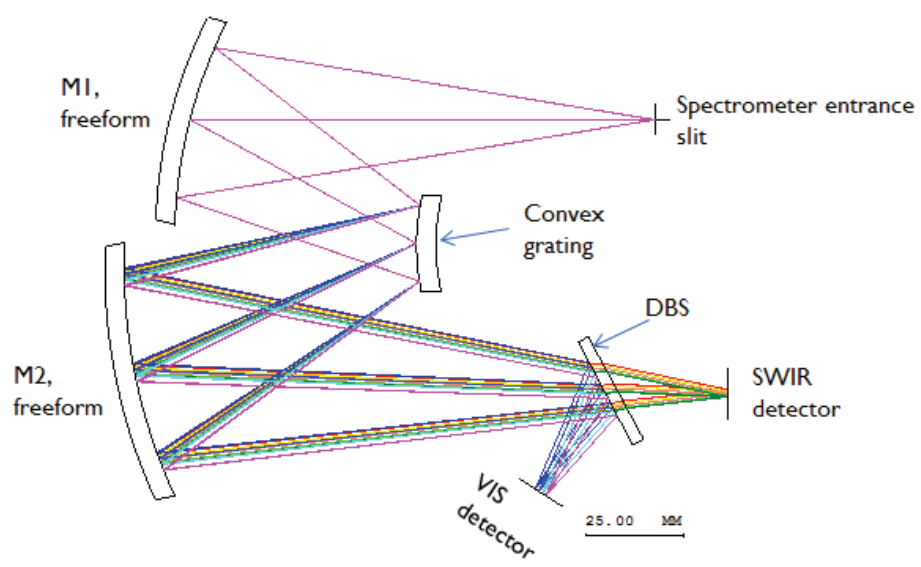

Figure 9. spectrometer for the second instrument option: 2 freeform mirrors, spherical convex grating with period $35 \mu \mathrm{m}$.

Separation VIS/SWIR with a dichroic plate. Spectrometer volume is 1.5 liters 
The spectral resolution is $10 \mathrm{~nm}$ over SWIR band and $5 \mathrm{~nm}$ over VNIR band. VNIR SNR is thus computed using binning over two spectral samples. It may be interes ting to have access to this improved spectral res olution of $5 \mathrm{~nm}$, even with degraded SNR.

The figure below gives a view of the telescope and the Offner spectrometer:

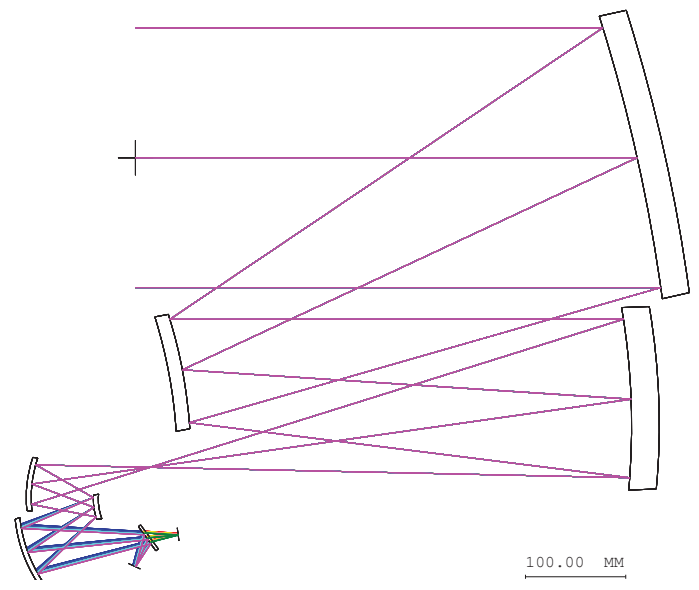

Figure 10. telescope + spectrometer for the second instrument option

\subsection{Instrument with two grating spectrometers}

To further improve compactness, and thus grating diffraction efficiency another option is to use two spectrometers, with two gratings: one spectrometer dedicated to VIS ([400-1000]nm), and one spectrometer dedicated to SW IR wavelengths ([1000-2500]nm). It is possible to drastically improve gratings' diffraction efficiency:

\section{Grating optimization:}

Grating for VIS spectrometer ([400-1000]nm):

A monoblazed grating calculated the wavelength 580nm gives:

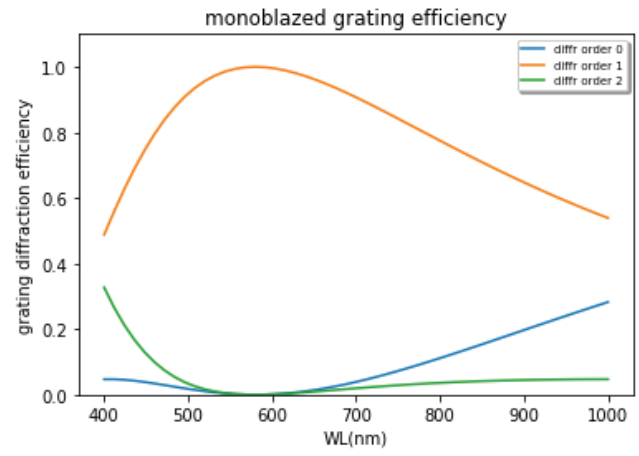

Figure 11. Diffraction efficiency with scalar model of a monoblazed grating over the [400-1000nm] spectral band. Blazed wavelength is $580 \mathrm{~nm}$. 
A few trials showed that it is not possible to get a better efficiency with the use of a multiblazed grating. Besides it is better to use a classical monoblazed grating if possible, for cost reasons.

Grating for SWIR spectrometer ([1000-2500]nm):

Multiblazed profile is necessary so that grating diffraction efficiency gets higher for wavelengths with the smallest luminance (the longer the wavelength, the larger the diffraction efficiency shall be).

Multiblazed grating profile, and the related diffraction efficiency is given in the plots below:
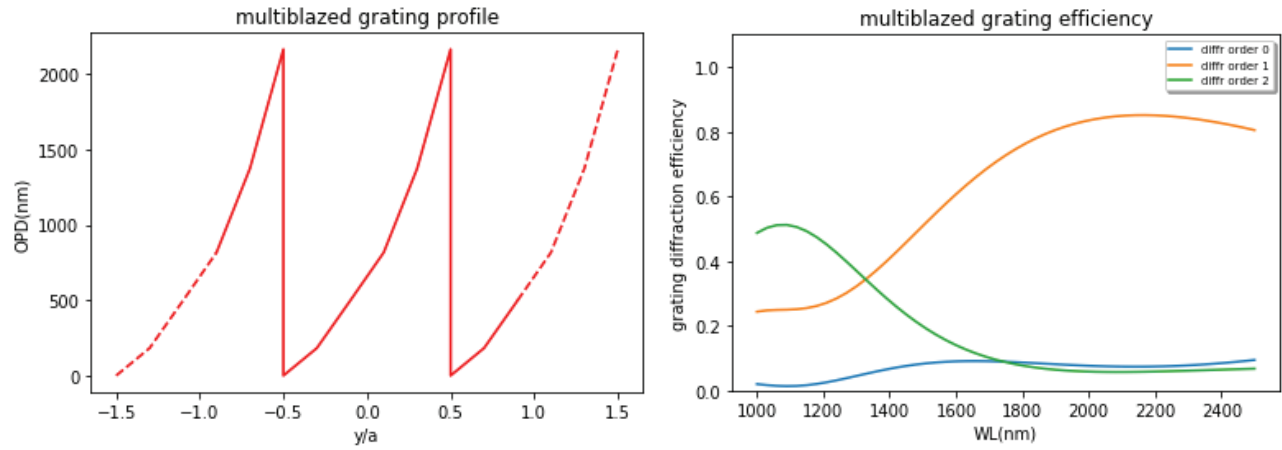

Figure 12. Multiblazed grating profile optimized for the [1000,2500nm] spectral band. On the right: Diffraction efficiency for this multiblazed grating. The five wavelengths used for the multiblazed profile are: $[911,1579,1590,2784,3953] \mathrm{nm}$

\section{Optical design:}

Splitting can be implemented at s lit level, with spectral splitting using a Dichroic Beam Splitter or with spatial splitting using two slits in the teles cope focal plane. The « in-the-field » splitting increases telescope ALT field of view, but saves a dichroic beam splitter and its related drop of transmission. Polarization sensitivity is also improved: this option is preferred.

Table 4. The instrument key parameters are calculated from SNR budget and taking into account the grating efficiency:

\begin{tabular}{|l|r|r|r|r|}
\hline wavelength $(\mathrm{nm})$ & 600 & 860 & 1600 & 2200 \\
\hline grating efficiency & 0.90 & 0.69 & 0.61 & 0.85 \\
\hline instrument transmission & 0.53 & 0.41 & 0.36 & 0.50 \\
\hline Lref $(\mathrm{W} / \mathrm{m} 2 / \mu \mathrm{m} / \mathrm{sr})$ & 70 & 70 & 15 & 5 \\
\hline EPD $(\mathrm{mm})\left(^{*}\right)$ & \multicolumn{5}{|c|}{236} \\
\hline SNR & OK & OK & OK & OK \\
\hline
\end{tabular}

With these data and some margin, instrument dimensioning gives Entrance Pupil Diameter of 236mm to reach the SNR specification at $2200 \mathrm{~nm}$.

The telescope design is similar to the 2 cases described before. The volume is optimized, thanks to a smaller entrance pupil size.

Telescope volume is 50 liters 
The gap between the two slits is set to $1.5 \mathrm{~mm}$ in order to ease separation of the 2 sub wavebands.

The spectrometers are Dys on spectrometers: as we need two such spectrometers the Dyson has the advantage to offer the smallest volume, and the smallest number of optical components.

Each Dyson spectrometer includes a silica plano-convex lens, with an as pherical convex face, and a concave grating.

Table 5. VIS and SWIR Dyson spectrometers characteristics:

\begin{tabular}{|l|l|l|}
\hline & VIS spectrometer & SWIR spectrometer \\
\hline grating & $\begin{array}{l}\text { Substrate: } \text { Spherical shape, concave } \\
\text { Radius of curvature }=143 \mathrm{~mm} \\
\text { Grating period }=49.5 \mu \mathrm{m}\end{array}$ & $\begin{array}{l}\text { Substrate: } \text { Spherical shape, concave } \\
\text { Radius of curvature }=165 \mathrm{~mm} \\
\text { Grating period }=62 \mu \mathrm{m}\end{array}$ \\
\hline Spectrometer length & $150 \mathrm{~mm}$ & $160 \mathrm{~mm}$ \\
\hline
\end{tabular}

The SWIR spectrometer tends to be longer than the VIS spectrometer, due to the larger waveband it covers.

Figure below depicts the optical layout of the whole instrument for this concept:
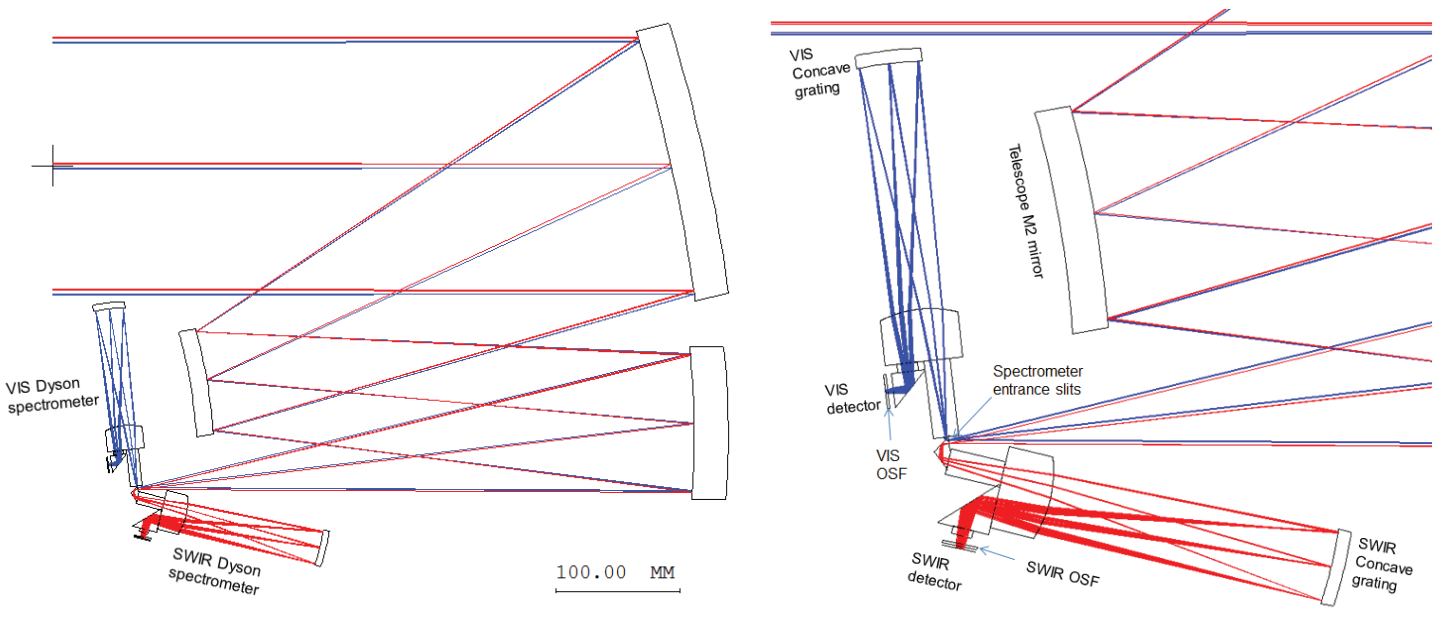

Figure 13. instrument for the third option: one telescope and two Dyson spectrometers for the VIS \& SWIR sub spectral bands. On the right: zoom on the spectrometers

The 2 wavebands are split by using small prisms after the slit plane. The SWIRpath is thus folded, in order to optimize the instrument global volume:

OSF (Order Sorting Filters) are implemented in front of each detector. 


\section{CONCLUSION:}

Hyperspectral ins truments with a large spectral band and using a grating spectrometer have to deal with the diffraction efficiency of the grating, which is the main driver for the instrument volume and compactness optimization. A low diffraction efficiency grating implies a large telescope pupil: the size of the instrument is driven by the telescope size rather than by the spectrometer.

For instruments covering VIS to SWIR spectral bands we demonstrate the interest to split the spectral band into 2 spectrometers, with dedicated and customized grating profiles. Grating efficiency is maximized, leading to a smaller instrument.

Besides we demonstrate that for this class of application, where instrument volume is driven by the telescope volume, a classical, spherical substrate for the grating permits a good compactness. Using a freeform substrate is not necessary.

Table 6. comparison of the three options

\begin{tabular}{|l|l|l|l|l|}
\hline Solution & description & $\begin{array}{l}\text { entrance pupil } \\
\text { diameter (mm) }\end{array}$ & $\begin{array}{l}\text { Number of } \\
\text { detectors }\end{array}$ & $\begin{array}{l}\text { Telescope volume } \\
\text { (liters) }\end{array}$ \\
\hline 1 & $\begin{array}{l}\text { common spectrometer, 1st } \\
\text { diffraction order }\end{array}$ & 350 & 1 & 100 \\
\hline 2 & $\begin{array}{l}\text { common spectrometer, } \\
\text { diffraction order 1 and 2 }\end{array}$ & 300 & 2 & 81 \\
\hline 3 & $\begin{array}{l}\text { dedicated spectrometer to } \\
\text { each sub waveband }\end{array}$ & 236 & 2 & 50 \\
\hline
\end{tabular}

\section{REFERENCES}

[1] J. Dyson, "Unit magnification optical system without Seidel aberrations," J. Opt. Soc. Am. 49(7), 713-716 (1959).

[2] L. Mertz, "Concentric spectrographs," Appl. Opt. 16(12), 3122-3124 (1977).

[3] A. Offner, "New concepts in projection mask aligners," Opt. Eng. 14(2), 130-132 (1975).

[4] B. Van Gorp, P. Mouroulis, D.W. Wilson, R.O. Green, "Design of the compact wide swath imaging spectrometer (CW IS),” Proc. SPIE, 9222, 92220C (2014). 